\title{
Complete cure of advanced hepatocellular carcinoma with right adrenal gland metastasis and portal vein thrombosis by multiple applications of an interdisciplinary therapy: Case report with 8-year follow up
}

\author{
Hojung Jung ${ }^{1}$, Byung Ik Kim¹, Yong Kyun Cho', Woo Kyu Jeon', Hong Joo Kim', and Hyun Pyo Hong ${ }^{2}$ \\ 'Division of Gastroenterology, Department of Internal Medicine and ${ }^{2}$ Radiology, Kangbuk Samsung Hospital, Sungkyunkwan University \\ School of Medicine, Seoul, Korea
}

Hepatocellular carcinoma (HCC) is the sixth most common cause of death worldwide and the main cause of primary liver cancer. The principle problem of HCC is the poor prognosis, since advanced HCC reportedly has a median survival of only 9 months. The standard therapies are sorafenib and regorafenib, but the outcomes remain unclear. We report a 60 -year-old man with advanced HCC with right adrenal gland metastasis and portal vein tumor thrombosis, who showed a complete response to multiple applications of an interdisciplinary therapy. (Clin Mol Hepatol 2018;24:424-429)

Keywords: Hepatocellular carcinoma; Adrenal gland; Portal vein thrombosis

\section{INTRODUCTION}

Hepatocellular carcinoma $(\mathrm{HCC})$ is the 6th most common cause of cancer and the second most common cause of cancer-related death worldwide.' Incidence data from 1999 to 2014 were collected from the Korea National Cancer Incidence Database. ${ }^{2}$ Estimated 15,593 new cases and 1,057 deaths are occurred during the period of study. ${ }^{3}$ However, the main problem is a poor prognosis of HCC. A median survival of advanced HCC is 9 months, and Sorafenib is the first approved systemic therapy for advanced $\mathrm{HCC}^{4}$ And regorafenib is approved by Food and Drug Administration (FDA) on April 27, 2017. In this study, we report a case of a 60-year-old man with advanced HCC with right adrenal gland metastasis and portal vein tumor thrombosis who achieved complete response after multiple and interdisciplinary therapies, maintained recurrence free for 8 years of follow up.

\section{CASE REPORT}

A 60-year-old man visited the hospital with febrile sense over the prior 7 days. He had no past history of relevant medical record. He socially consumed alcohol about 1-2 times for month. Laboratory tests revealed a white blood cell count of $5,500 / \mu \mathrm{L}$, with $70.1 \%$ neutrophils, a platelet level of $160,000 / \mu \mathrm{L}$, and prothrombin time (international normalized ratio, INR) of 1.10. The

\section{Abbreviations:}

CT, computed tomography; FDA, Food and Drug Administration; HCC, hepatocellular carcinoma; PET-CT, positron emission tomography-computed tomography; PVTT, portal vein tumor thrombosis; TACE, transarterial chemoembolization

\author{
Corresponding author : Byung Ik Kim \\ Division of Gastroenterology, Department of Internal Medicine, Kangbuk \\ Samsung Hospital, Sungkyunkwan University School of Medicine, 29 \\ Saemunan-ro, Jongno-gu, Seoul 03181, Korea \\ Tel: +82-2-2001-8553, Fax: +82-2-736-8360 \\ E-mail: bik.kim@samsung.com \\ https://orcid.org/0000-0002-7215-7715
}



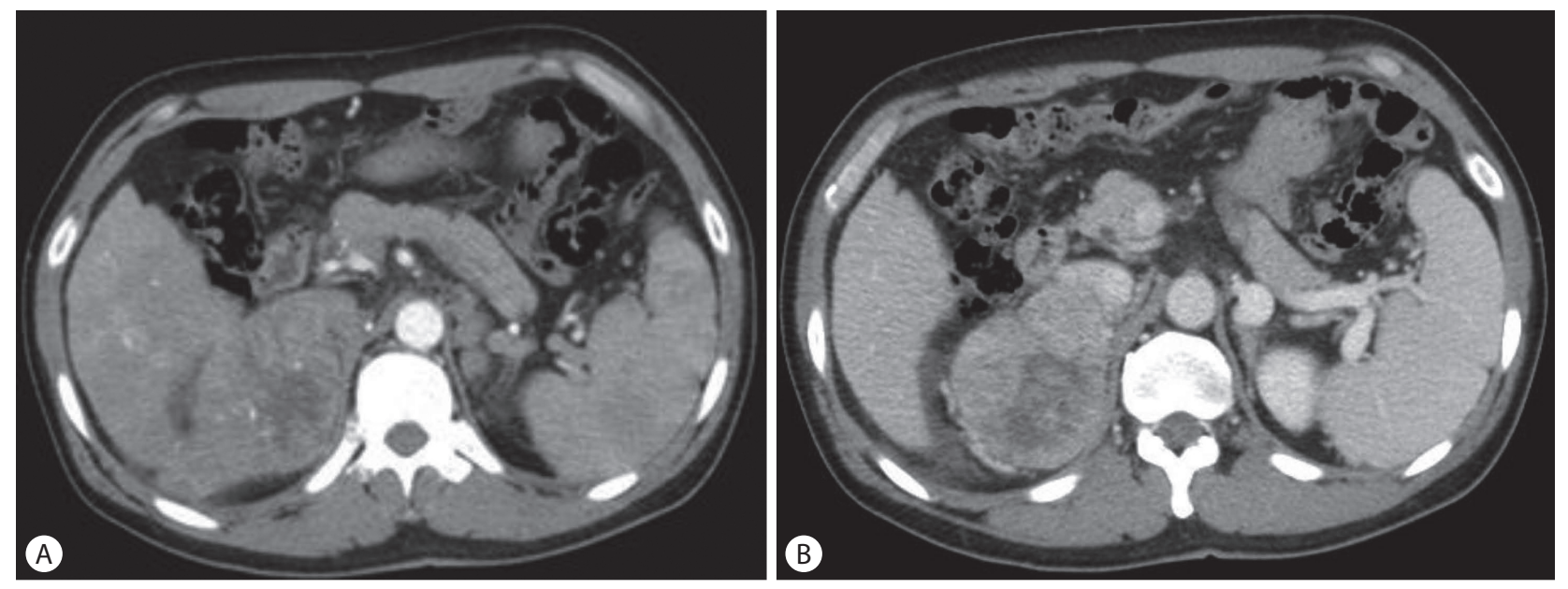

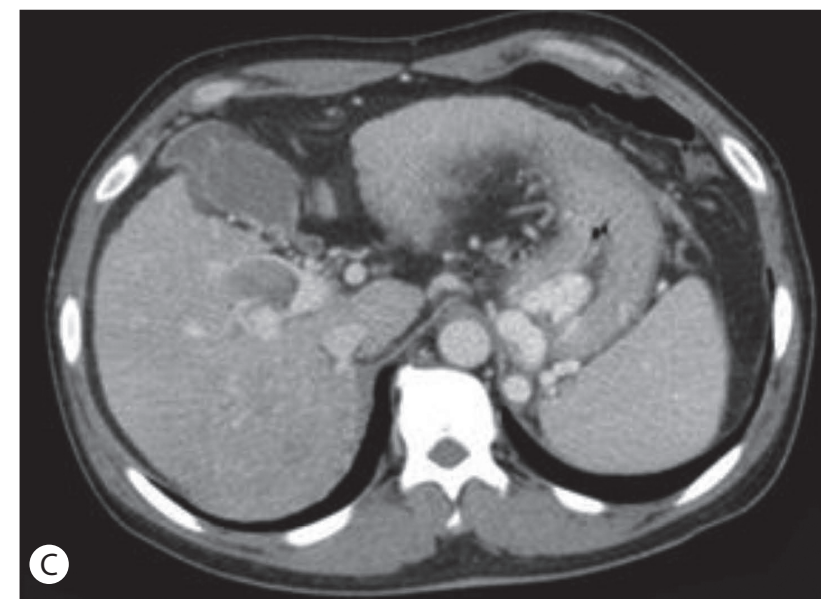

patient's blood chemistry profile was as follows: fasting glucose $88 \mathrm{mg} / \mathrm{dL}$; total protein, $7.6 \mathrm{~g} / \mathrm{dL}$; albumin, $3.6 \mathrm{~g} / \mathrm{dL}$; total bilirubin, $0.83 \mathrm{mg} / \mathrm{dL}$; aspartate aminotransferase, $96 \mathrm{U} / \mathrm{L}$; alanine aminotransferase, $104 \mathrm{U} / \mathrm{L}$; alkaline phosphatase, $189 \mathrm{U} / \mathrm{L}$; gamma-glutamyl transferase, $101 \mathrm{U} / \mathrm{L}$. Serologic tests for tumor markers were as follows: alpha-fetoprotein, 1,200 ng/mL (normal range, 0-7); CA 19-9, $28.71 \mathrm{U} / \mathrm{mL}$ (0-37). Serologic tests for viral markers were as follows: HBs Ag, positive; $\mathrm{HBe} A g$, negative; hepatitis $B$ virus DNA 854,515 IU/mL; and anti-hepatitis C virus, negative.

A computed tomography (CT) scan of the abdomen revealed an approximately $8 \mathrm{~cm}$ sized huge ovoid shape heterogenous arterial enhancement and delayed wash out mass lesion. We observed a suggested HCC in the liver $56 / 7$ with direct invasion of right adrenal gland. Moreover, an approximately $3 \mathrm{~cm}$ sized arterial enhancing lesion and delayed wash out lesion were observed in the liver S8. This lesion invaded directly the right main portal vein and formed the tumor thrombus at right main portal vein. This result suggests HCC in the liver S6/7 with direct invasion on right portal
Figure 1. Liver computed tomography (CT) findings. An arterial-phase image revealed an enhanced mass of size $8.5 \times 8 \mathrm{~cm}$ at $56 / 7$ of the liver (A), while a delayed-washout image revealed an $8-\mathrm{cm}$ lesion at the same location (B). A tumor thrombus was noticed in the right main portal vein (C).

vein (Fig. 1). One $\mathrm{cm}$ sized lymph node was noticed at porta hepatis. A positron emitting tomography-computed tomography (PETCT) scan noticed a $8.5 \mathrm{~cm}$ sized hypermetabolic mass in the right liver $56 / 7$, which directly invades the right adrenal gland. There were no significant increasing of abnormal Fludeoxyglucose uptake in scan (Fig. 2). Portal vein thrombus was not noticed on PET-CT scan at initial work up.

Considering the imaging test and elevation of tumor marker, we diagnosed HCC with invasion of right adrenal gland and right portal vein. Transarterial chemoembolization (TACE) was performed in October 2009. Large tumor staining revealed in right lobe of liver supplied by right hepatic artery and right phrenic artery. 2nd TACE was done at 2009.12. We considered radiotherapy about right adrenal gland mass. Radiotherapy (dose: 33cGy; number of session: 11 times) was performed for targeting liver $56 / 7 / 8$, right adrenal gland, and right portal vein during on month. We considered operation rather than radiofrequency ablation, because colon was near the suspicious lesion of subtle tumor staining. After first TACE, the right main portal vein thrombus was still found, al- 

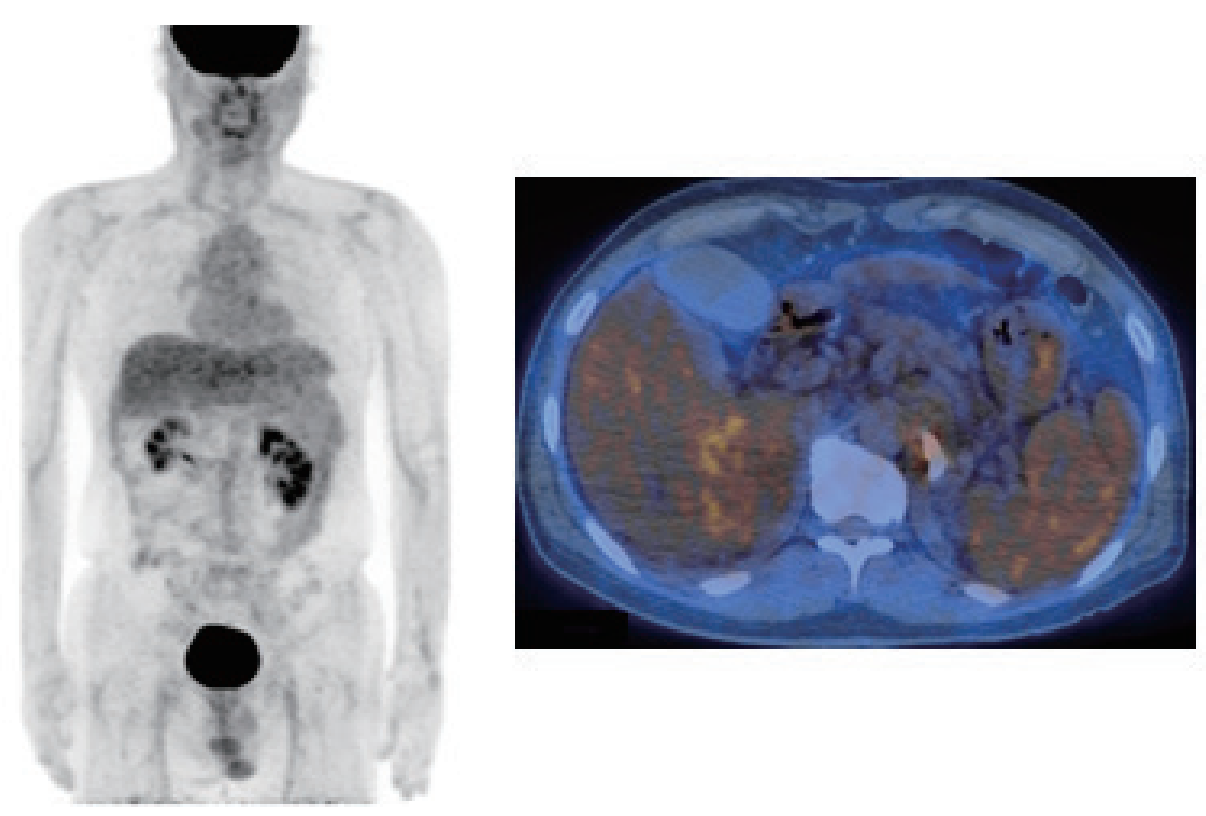

Figure 2. Combined positron-emission tomography and computed tomography revealed an $8.5-\mathrm{cm}$ hypermetabolic mass in the right liver at $56 / 7$ that directly invaded the right adrenal gland.
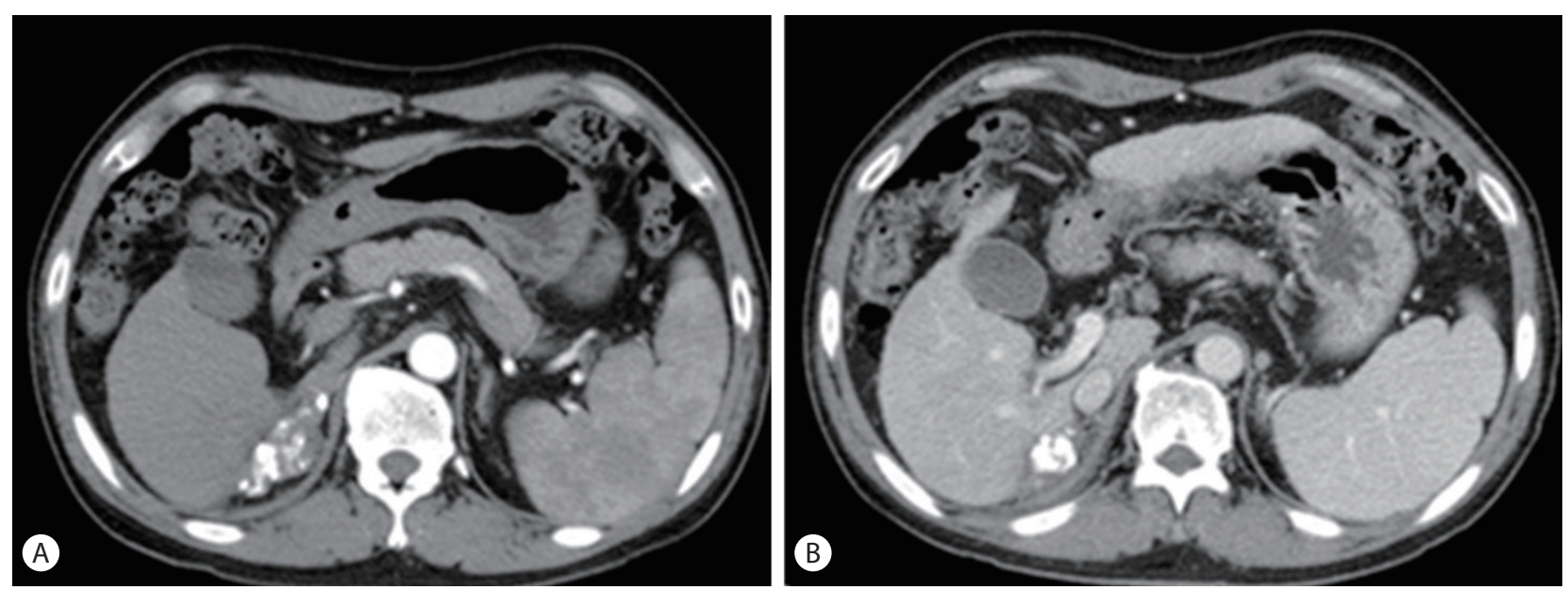

Figure 3. Computed tomography of the liver showed a lesion with an increased Lipiodol uptake and decreased tumor volume (A). The tumor thrombus was not observed in the right main portal vein (B).

though they were reduced in size for $1 \mathrm{~cm}$. But no tumor staining was found in the right main portal vein after second TACE. We conducted CT for follow up, after third TACE. CT scans were performed to rule out the recurrence or progression at March 2010 and July 2010. Portal vein thrombosis was also not observed in CT scan after radiotherapy and third TACE (Fig. 3). Therefore, we concluded that the portal vein thrombosis observed at baseline CT was a tumor thrombosis.

Before the operation, We examined the PET-CT to rule out distance metastasis. There was no significant increase in abnormal fludeoxyglucose uptake from the scan. Laboratory tests revealed a white blood cell count of 5,400/uL, with $60.6 \%$ neutrophils, a platelet level 106,000 of $/ \mu \mathrm{L}$, and INR 1.12. The patient's blood chemistry profile was as follows: albumin, $4.4 \mathrm{~g} / \mathrm{dL}$; total bilirubin, $0.65 \mathrm{mg} / \mathrm{dL}$; aspartate aminotransferase, $31 \mathrm{U} / \mathrm{L}$; alanine aminotransferase, $31 \mathrm{U} / \mathrm{L}$. Therefore, we concluded the patient's status as stable disease. The patient was reluctant to undergo surgery, however after our medical team's advice, operation was performed on patient consent.

Hepatic segmentectomy (S6/7), cholecystectomy and right adrenal resection was performed at January 20, 2011. Dissection was difficult because of severe adhesion after TACE and radiotherapy, but all suspicious gross tumor was resected. After the operation, Microscopic pathology of the resected liver, gallbladder, right ad- 

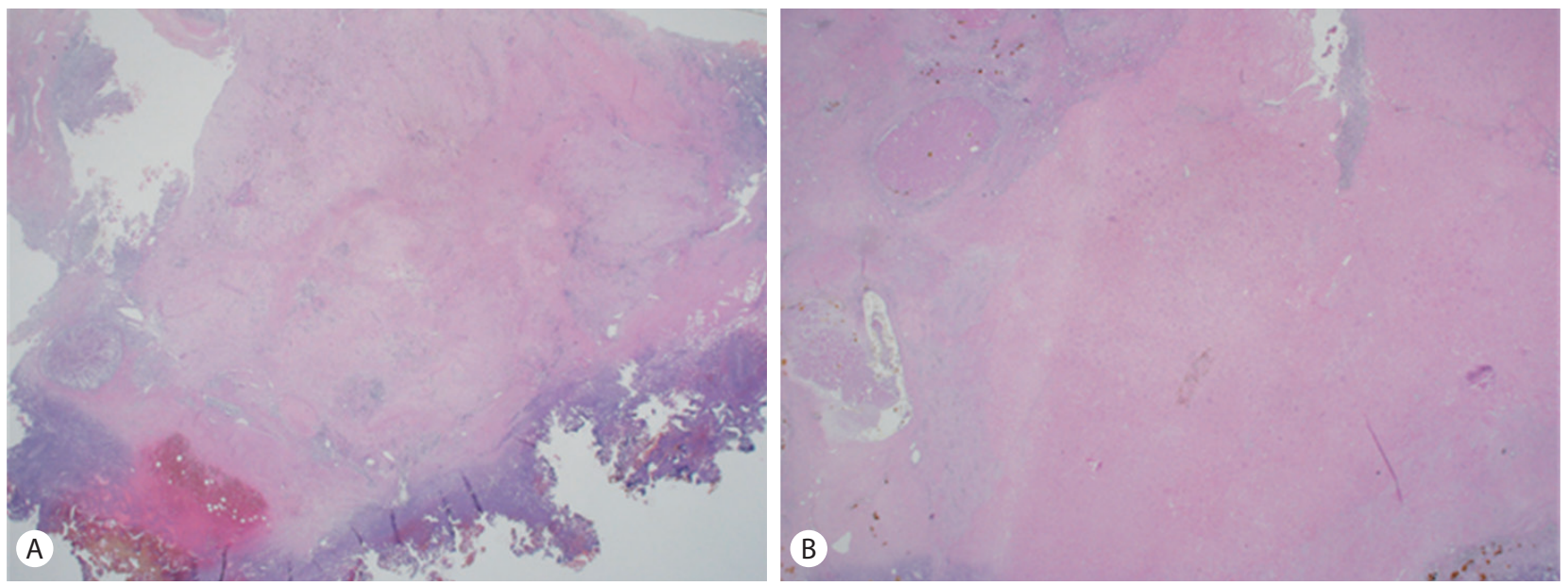

Figure 4. Microscopic pathology revealed hepatocellular carcinoma surrounding the adrenal gland (A). It was impossible to evaluate the tumor grading and pattern since the tumor was undergoing total necrosis after transarterial chemoembolization and radiotherapy (B).
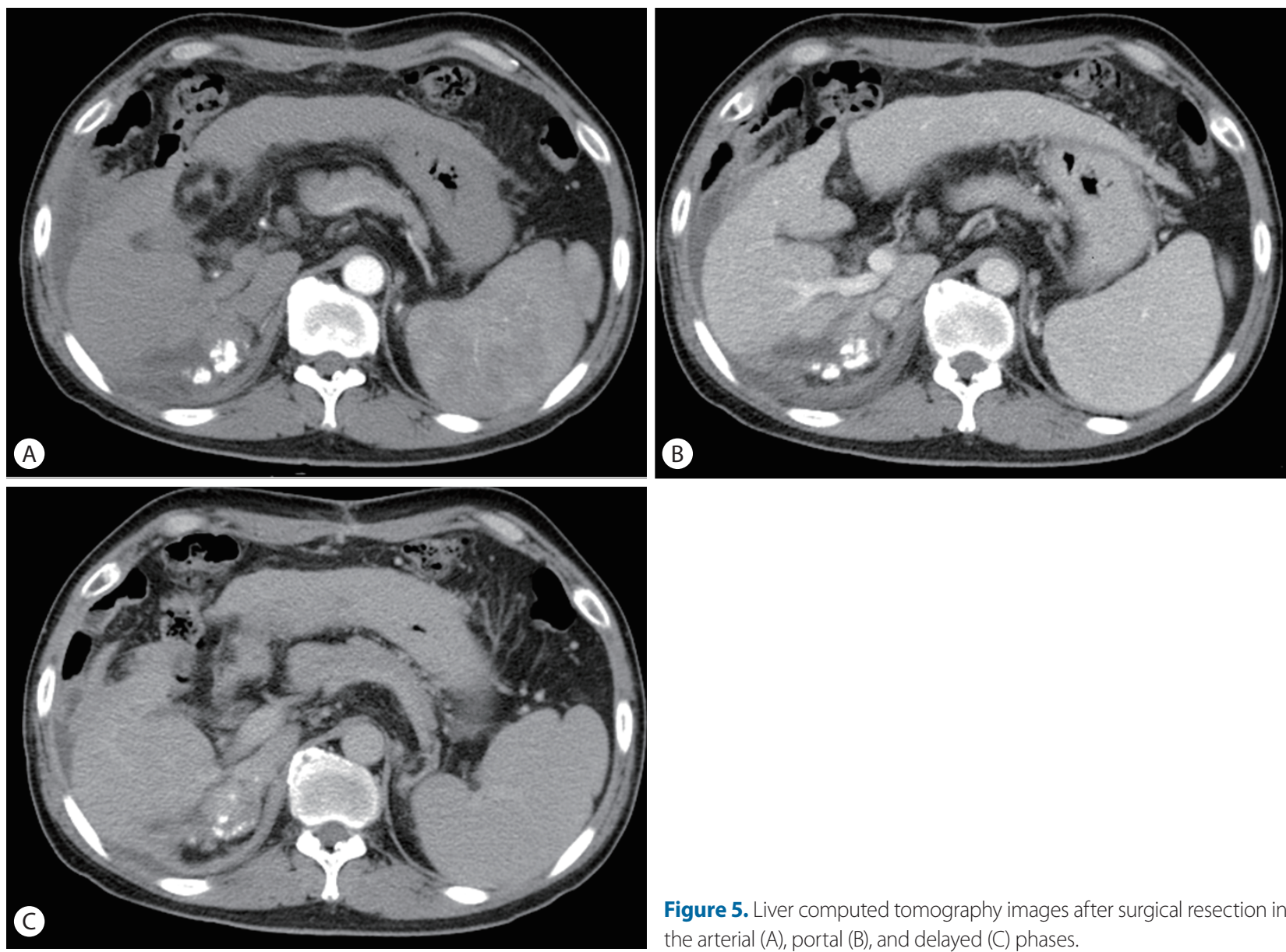

Figure 5. Liver computed tomography images after surgical resection in the arterial (A), portal (B), and delayed (C) phases.

renal gland, and right adrenal LN shown ischemic necrosis and fibrosis formation without tumor cell. There were revealed necrosis and fibrosis changes without malignancy (Fig. 4). Partial lipiodol laden mass was removed after operation at liver $\$ 6 / 7$ and right adrenal gland. However partial lipiodol laden mass was noticed at remnant right adrenal gland. We decided to follow up this re- 
mained partial lipiodol laden mass. Moreover, small amount of fluid collection was observed at operation site, which we considered as a postoperative change. There was no abnormal arterial enhancing lesion at CT imange study (Fig. 5). The patient was discharged from the hospital after surgery. Blood test revealed normalized alpha fetoprotein as $0.7 \mathrm{ng}$.

Laboratory tests for PIVKA-II were as follows: initial result, 495 $\mathrm{mAU} / \mathrm{mL}$; after third TACE, $20 \mathrm{mAU} / \mathrm{mL}$; brefore operation, 21 $\mathrm{mAU} / \mathrm{mL}$. The last check of PIVKA-II was revealed $20 \mathrm{mAU} / \mathrm{mL}$ at 2015.6. We underwent follow-up monitoring the patient with dynamic liver CT and tumor marker levels every 4-6 months. In recent study at January 2017, CT scan of liver revealed no recurrence and interval changes than last study. The patient completely cured and no evidence of recurrence with 8 year follow up.

\section{DISCUSSION}

HCC stage can be classified into a very early, early, intermediate, advanced, and end stage according to their size, number, ECOG performance, existence of portal vein tumor thrombosis (PVTT). ${ }^{5}$ According to the Barcelona Clinic Liver Cancer staging system, HCC with PVTT is classified as an advanced stage. Malignant portal vein thrombosis is the leading cause of poor prognosis, resulting in a median survival time of 2-4 months without treatment. As it is impossible to treat surgical resection, there are no other treatment methods than systemic chemotherapy. Sorafenib is the first approved systemic therapy and regorafenib was approved by FDA for advanced $\mathrm{HCC}^{6,7}$ And regorafenib is approved by FDA on April 27, 2017. Sorafenib has been acknowledged as a standard therapy in patients with advanced HCC, but it only provides limited survival benefits and treatment responses. ${ }^{8,9}$ In the clinical fields, hepatologists are considering to find other treatment methods, other than sorafenib.

According to a review article of non-operative Treatments for HCC with PVTT in Journal of Clinical and Translational Hepatology, the median survival times is 0.78 years $(95 \% \mathrm{Cl}, 0.70-0.83)$ in patients of HCC accompanied by right PVTT (Vp3). The median survival times of Vp3 patients in the TACE and supportive care group was 11.0 and 1.43 months, respectively $(P<0.01)$, indicating that the TACE had a significant longer survival than the supportive treatment. $^{10}$

According to case report on Gastroenterology, a 56 year old man with HCC accompanied by PVTT who achieved complete remission for 8 years after receiving conventional TACE. ${ }^{11}$ Herein, we report a case of a 60 year-old man with HCC accompanied by metastasis of Rt. adrenal gland and PVTT, who showed a complete remission for 8 years after multiple and interdisciplinary therapy, and maintained recurrence free for 8 years of follow up. Due to the patient's conserved liver function, and a good ECOG performance, we conducted other options than sorafenib. As in this case, if the patients have a positive liver function, we can consider other treatment options such as TACE, radiotherapy, and operation over sorafenib. Therefore, we suggest HCC may be cured completely even with an advanced stage with other treatment options and combination therapy rather than monotherapy.

\section{Authors' contribution}

Authors give final approval of the version to be submitted and any revised version.

\section{Financial support}

This work was supported in part by a Samsung Biomedical Research Institute grant and Medical Research Funds from Kangbuk Samsung Hospital (Seoul, Korea).

\section{Conflicts of Interest}

The authors have no conflicts to disclose.

\section{REFERENCES}

1. Ferlay J, Soerjomataram I, Dikshit R, Eser S, Mathers C, Rebelo M, et al. Cancer incidence and mortality worldwide: sources, methods and major patterns in GLOBOCAN 2012. Int J Cancer 2015;136:E359386.

2. Jung KW, Won YJ, Oh CM, Kong HJ, Cho H, Lee JK, et al. Prediction of cancer incidence and mortality in Korea, 2016. Cancer Res Treat 2016;48:451-457.

3. Siegel RL, Miller KD, Jemal A. Cancer statistics, 2016. CA Cancer J Clin 2016;66:7-30.

4. Allaire M, Nault JC. Advances in management of hepatocellular carcinoma. Curr Opin Oncol 2017;29:288-295.

5. Gomaa Al, Waked I. Recent advances in multidisciplinary management of hepatocellular carcinoma. World J Hepatol 2015;7:673-687.

6. Kim DW, Talati C, Kim R. Hepatocellular carcinoma (HCC): beyond sorafenib-chemotherapy. J Gastrointest Oncol 2017;8:256-265.

7. Llovet JM, Ricci S, Mazzaferro V, Hilgard P, Gane E, Blanc JF, et al. Sorafenib in advanced hepatocellular carcinoma. N Engl J Med 2008;359:378-390.

8. Keating GM, Santoro A. Sorafenib: a review of its use in advanced hepatocellular carcinoma. Drugs 2009;69:223-240. 
Hojung Jung, et al. Complete cure of advanced HCC

9. Lu LC, Chen PJ, Yeh YC, Hsu CH, Chen HM, Lai MS, et al. Prescription patterns of sorafenib and outcomes of patients with advanced hepatocellular carcinoma: a national population study. Anticancer Res 2017;37:2593-2599.

10. Moriguchi M, Furuta M, Itoh Y. A review of non-operative treatments for hepatocellular carcinoma with advanced portal vein tumor thrombus. J Clin Trandl Hepatol 2017;5:177-183.

11. Kim SB. Diffuse infiltrative hepatocellular carcinoma with portal vein tumor thrombosis completely cured by transcatheter arterial chemoembolization: case report with 8-year follow-up. Case Rep Gastroenterol 2016;10:623-628. 